\title{
Enzymatic propyl gallate synthesis in solvent-free system: Optimization by response surface methodology
}

\author{
Ahlem Bouaziz ${ }^{\mathrm{a}, 1}$, Habib Horchani ${ }^{\mathrm{a}, 1}$, Nadia Ben Salem $^{\mathrm{a}}$, Ali Chaari ${ }^{\mathrm{a}}$, \\ Moncef Chaabouni ${ }^{\mathrm{b}}$, Youssef Gargouri ${ }^{\mathrm{a}}$, Adel Sayari ${ }^{\mathrm{a}, *}$ \\ a Laboratoire de Biochimie et de Génie Enzymatique des Lipases, ENIS, BP1173, Sfax 3038, Tunisia \\ ${ }^{b}$ Laboratoire de Chimie Industrielle, ENIS, BP1173, Sfax 3038, Tunisia
}

\section{A R T I C L E I N F O}

\section{Article history:}

Received 22 June 2010

Received in revised form 21 August 2010

Accepted 27 August 2010

Available online 8 October 2010

Keywords:

Immobilized Staphylococcus xylosus lipase

Enzymatic synthesis

Response surface methodology

Propyl gallate

Antioxidant

Antibacterial

\begin{abstract}
A B S T R A C T
The ability of a non-commercial immobilized Staphylococcus xylosus lipase to catalyze the esterification of propanol with gallic acid was investigated and the antioxidant as well as the antimicrobial activities of the ester formed were evaluated. The response surface methodology, based on a three variables Box-Behnken design (reaction temperature, enzyme amount and 1-propanol/gallic acid molar ratio), was used to optimize the experimental conditions of propylgallate synthesis. The maximum conversion yield $(90 \% \pm 3.5)$ was obtained by using $400 \mathrm{IU}$ of immobilized lipase and a propanol/gallic acid at a molar ratio of 160 at $52{ }^{\circ} \mathrm{C}$. The obtained ester was characterized by spectroscopic methods, NMR and FTIR. The antioxidant activity of propyl gallate was evaluated and compared to the synthetic classical antioxidants, BHA and ascorbic acid, taken as references. In addition, the antimicrobial activity of the propyl gallate was tested against S. xylosus, Escherchia coli and Staphylococcus aureus using disc diffusion and macrodilution methods. Our results show that the synthesized propyl gallate ester presents a higher antioxidant and antimicrobial power than the parent gallic acid as well as the synthetic classical antioxidants.
\end{abstract}

(C) 2010 Elsevier B.V. All rights reserved.

\section{Introduction}

Antioxidants belong to a specific class of chemical compounds that have the ability to prevent or to reduce the rate of oxidative reactions of substrates in food, pharmaceuticals, nutraceuticals and other consumer goods. These compounds, such as ascorbic acid, are either naturally present or deliberately added. The addition of artificial antioxidants to maintain food quality and nutritional values has been routinely carried out for several decades in the food industry. This food treatment technology is particularly important in controlling the oxidation of lipids, which may result in the changes of the odour of edible oil and fat-containing food products and may turn them rancid. The mechanism of action of antioxidants is to interfere with the formation of free radicals that propagate oxidation [1]. Phenolic compounds that have unique chemical characteristics which form low-energy radicals through stable resonance hybrids are considered to be good antioxidants for food.

Abbreviations: SXL2, Staphylococcus xylosus lipase 2; SXL2 $2_{\mathrm{i}}$, immobilized Staphylococcus xylosus lipase 2; IU, Enzymatic International Unit; $\mathrm{CaCO}_{3}$, calcium carbonate; HPLC, High Performance Liquid Chromatography; BHA, butylated hydroxyanisole; PG, propyl gallate.

* Corresponding author. Tel.: +216 74675 055; fax: +216 74675055

E-mail address: adelsayari@yahoo.fr (A. Sayari).

1 They have participated equally to this work.
The common synthetic phenolic compounds used as food additives in many countries include butylated hydroxylanisole (BHA), butylated hydroxyltoluene (BHT), propyl gallate (PG), octyl gallate and dodecyl gallate. However, these antioxidants have been proved to exert different degrees of adverse and toxic effects upon consumption $[2,3]$. Thus to protect people, the quantity of these compounds present in food preparations is strictly controlled in many countries worldwide. The regulatory levels are generally based on the estimation of an acceptable daily intake for a healthy individual.

Propyl gallate (PG, propyl 3,4,5-trihydroxybenzoate) is used as antioxidant in processed food, cosmetics and food packing materials in order to prevent rancidity and spoilage. According to the US Food and Drug Administration list, PG is also used to preserve and stabilize medicinal preparations [4]. It is an allowed additive in the European Union and in many others countries. Because of its prevalent usage, the potential toxicity of PG has been investigated in vivo $[5,6]$ and in vitro to assess various toxicological properties, i.e. mutagenicity [7] and cytogenetic effects [8]. Although PG has a low toxicity, it has various effects on tissue and cell functions.

Several studies have demonstrated the benefits of PG as an antioxidant $[6,9,10]$, as a chemopreventive agent $[11,12]$ and as an anti-inflammatory agent [13]. For instance, PG is an efficient protector of liver cells from lipid peroxidation by oxygen radicals [6]. Furthermore, it is almost as effective as the vitamin E analogue, Trolox and it is even more effective than several water 


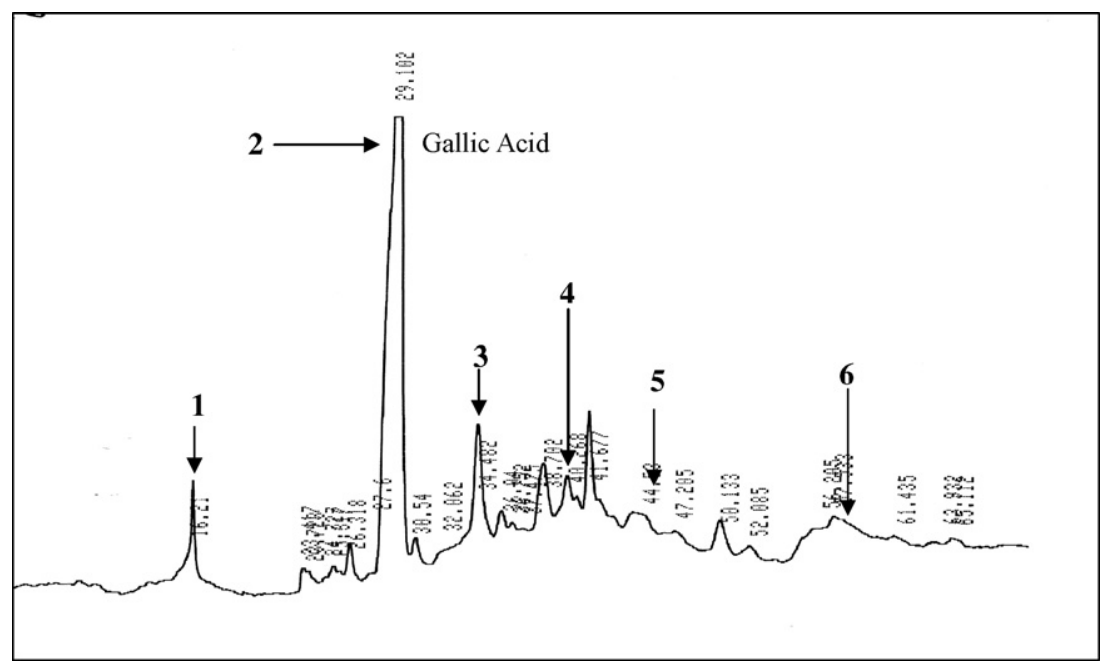

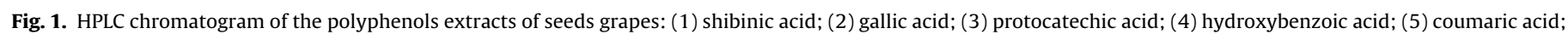
(6) cinnamic acid.

soluble antioxidants such as ascorbate [6]. Traditionally, this kind of compounds has been either isolated from natural sources or produced by chemical synthesis. However, the use of chemical process is being progressively restricted for many applications. Such later methods rely on the use of sophisticated experimental techniques and organic solvents which are prohibited for industrial applications, especially in food industries. The use of extracellular lipases as catalysts for esters production has a great potential. In fact, using a biocatalyst excludes the disadvantages of the chemical process by producing compounds which are highly pure with less or no downstream operations. Moreover, the use of a non-commercial lipase in an immobilized form would overcome the technological difficulties of production. Thus, it might be interesting to test the performance of new lipases in the synthesis of propyl gallate by direct esterification in solvent-free system. It is the purpose of this research to develop a non-commercial lipase-mediated process for the conversion of gallic acid and 1-propanol into propyl gallate. The effects of various reaction parameters (the lipase amount, the 1-propanol/gallic acid molar ratio, the reaction temperature) were optimized using response surface methodology. The antioxidant and the antibacterial activities of the propyl gallate were evaluated.

\section{Materials and methods}

\subsection{Reagents}

Whole grape seeds were collected in Tunisia and milled to a fine powder. Gallic acid and propyl gallate (98\% of purety) were purchased from Fluka (Buchs, Switzerland). The Hexane, chloroform, methanol were purchased from Prolabo (Paris, France). The calcium carbonate $\left(\mathrm{CaCO}_{3}\right)$ was obtained from Pharmacia (Uppsala, Sweden). Molecular sieve dehydrate with indicator for drying solvents $3 \AA$ A was purchased from Fluka (Buchs, Switzerland). All other used reagents are from analytical grade.

\subsection{Lipases}

\subsubsection{Production and immobilization of lipase}

Staphylococcus xylosus lipase was produced and immobilized onto $\mathrm{CaCO}_{3}$, as previously described [14].

\subsubsection{Lipase hydrolytic activity}

The lipase activity was titrimetrically measured with a pH-Stat (Metrohm, Herisau, Switzerland) under optimal conditions using olive oil emulsion as a substrate [15]. The activity was expressed as units per $\mathrm{mL}$ of enzymatic solution. One-unit (IU) of lipase activity corresponds to one $\mu \mathrm{mol}$ of fatty acid released per min at $\mathrm{pH} 8.5$ and at $55^{\circ} \mathrm{C}$.

\subsection{Polyphenols extraction}

Polyphenols were extracted, isolated and purified according to Nawaz et al. with a slight modification [16]. Ethanol, mixed with water, was selected as the best organic solvent used to improve the solubility of the bioactive components [17]. The maximum extraction yield was obtained when using a mixture of $50 \%$ of ethanol and $50 \%$ of water $(\mathrm{v} / \mathrm{v})$. The following protocol was used to the sample preparation. About $40 \mathrm{~g}$ of milled grape seeds were mixed with $200 \mathrm{~mL}$ of $50 \%$ ethanol solution and let for $1 \mathrm{~h}$ in the dark. The top phase was filtered through Whatman filter paper and the residue was resuspended in $150 \mathrm{~mL}$ of $95 \%$ of ethanol solution. The latter extraction step was repeated three times in order to extract the total polyphenols which are present in the sample. It's Noteworthy that during the first extraction, the majority of the polyphenols were removed. The identification of polyphenols and the purification of gallic acid were carried out by HPLC (Fig. 1). The concentrations were determined by calculating the HPLC peak areas which are proportional to the amount of analytes in a peak and presented as the mean of two determinations which were highly repeatable. Six compounds were identified (cinnamic, coumaric, protocatechic, shibinic, hydroxybenzoic and gallic acid) on the basis of their retention time compared to those considered as standards (Sigma Aldrich, 99\% puriss). One can note from Fig. 1 that gallic acid is the major compound detected. It represents more than $90 \%$ of the total polyphenols extracted from grape seeds. About $2 \pm 0.2 \mathrm{mg}$ of purified gallic acid were obtained from $1 \mathrm{~g}$ of grape seeds. The purified gallic acid was used to synthesize propylgallate.

\subsection{Enzymatic esterification}

The propyl gallate was obtained by the esterification of gallic acid with 1-propanol using immobilized S. xylosus lipase.

The esterification reactions were carried out in screw-capped flasks at various 1-propanol/gallic acid molar ratios (100-160) and with variable amounts of immobilized lipase (100-160 IU). The reaction mixture was incubated in an orbital shaker (Certomat $\mathrm{H} / \mathrm{HK}$, Germany, Melsungen) at various temperatures $\left(45-55^{\circ} \mathrm{C}\right)$ at $200 \mathrm{rpm}$. 
Table 1

Range of variables for the experimental design.

\begin{tabular}{lrrr}
\hline Variables & \multicolumn{2}{l}{ Levels } & \\
\cline { 2 - 4 } & \multicolumn{1}{c}{-1} & \multicolumn{1}{c}{0} & +1 \\
\hline$X_{1}$ : temperature $\left({ }^{\circ} \mathrm{C}\right)$ & 45 & 50 & 55 \\
$X_{2}:$ enzyme amount $(\mathrm{UI})$ & 100 & 300 & 500 \\
$X_{3}:$ 1-propanol/gallic acid molar ratio & 100 & 130 & 160 \\
\hline
\end{tabular}

A control without enzyme was run in parallel under the same conditions. Aliquots of the reaction mixture were periodically withdrawn. The immobilized enzyme was removed by centrifugation at $8000 \mathrm{rpm}$ for $5 \mathrm{~min}$. The residual acid content was determined by titrating with $0.07 \mathrm{~N}$ sodium hydroxide using phenolphthalein solution as an indicator and $3 \mathrm{~mL}$ of ethanol. The conversion yield of propyl gallate ester was calculated on the basis of the amount of the consumed acid [18].

\subsection{Experimental design}

The optimization of the experimental conditions of a propyl gallate synthesis was achieved by using the Response Surface Methodology (RSM) [19]. In this work, a quadratic polynomial model was set up in order to study the empirical relationship between conversion yield and three controlled factors namely: $X_{1}$, temperature; $X_{2}$, amount of immobilized lipase and $X_{3}, 1$ propanol/gallic acid molar ratio. In this part of the paper, we briefly discuss the principles governing the construction and analysis of the experimental design. To estimate the model coefficients, a three-variable Box-Behnken design, which requires 16 experiments (Table 1), is carried out in the cubic experimental domain. The experimental points are located in the middle of a cube ridges (12 experiments) and at the center of the cube (4 experiments) $[20,21]$. The model parameters are estimated by a least square fitting of the model to experimental results obtained in the design points. The adequacy of the model is tested using four check points [21,22].

Table 1 shows the selected variable levels. Table 2 shows the three-variable replicate Box-Behnken design used for fitting the following second-order mathematical model.

$$
\begin{aligned}
\hat{y}= & b_{0}+b_{1} X_{1}+b_{2} X_{2}+b_{3} X_{3}+b_{11} X_{12}+b_{22} X_{22}+b_{33} X_{32} \\
& +b_{12} X_{1} X_{2}+b_{13} X_{1} X_{3}+b_{23} X_{2} X_{3}
\end{aligned}
$$

Table 2

\begin{tabular}{|c|c|c|c|c|c|}
\hline \multirow[t]{2}{*}{ Run } & \multicolumn{3}{|c|}{ Variable values } & \multicolumn{2}{|c|}{ Esterification yield } \\
\hline & $X_{1}$ & $X_{2}$ & $X_{3}$ & Experimental & Predicted \\
\hline 1 & $-1(45)$ & $-1(100)$ & $0(130)$ & 35.40 & 38.17 \\
\hline 2 & 1(55) & $-1(100)$ & $0(130)$ & 49.30 & 50.17 \\
\hline 3 & $-1(45)$ & $1(500)$ & $0(130)$ & 77.90 & 77.02 \\
\hline 4 & 1(55) & $1(500)$ & $0(130)$ & 82.00 & 79.22 \\
\hline 5 & $-1(45)$ & $0(300)$ & $-1(100)$ & 69.10 & 66.42 \\
\hline 6 & 1(55) & $0(300)$ & $-1(100)$ & 74.30 & 73.52 \\
\hline 7 & $-1(45)$ & $0(300)$ & $1(160)$ & 78.70 & 79.47 \\
\hline 8 & 1(55) & $0(300)$ & 1(160) & 83.90 & 86.57 \\
\hline 9 & $0(50)$ & $-1(100)$ & $-1(100)$ & 42.60 & 42.5 \\
\hline 10 & $0(50)$ & $1(500)$ & $-1(100)$ & 73.80 & 77.35 \\
\hline 11 & $0(50)$ & $-1(100)$ & $1(160)$ & 60.00 & 56.45 \\
\hline 12 & $0(50)$ & $1(500)$ & $1(160)$ & 89.40 & 89.50 \\
\hline 13 & $0(50)$ & $0(300)$ & $0(130)$ & 82.00 & 83.67 \\
\hline 14 & $0(50)$ & $0(300)$ & $0(130)$ & 85.30 & 83.67 \\
\hline 15 & $0(50)$ & $0(300)$ & $0(130)$ & 82.70 & 83.67 \\
\hline 16 & $0(50)$ & $0(300)$ & $0(130)$ & 84.70 & 83.67 \\
\hline 17 & -0.5774 & -0.3333 & -0.2357 & 64.91 & 69.98 \\
\hline 18 & 0.5774 & 0.3333 & -0.2357 & 72.76 & 75.02 \\
\hline 19 & 0 & 0.6667 & -0.2357 & 82.64 & 86.23 \\
\hline 20 & 0 & 0 & -0.7071 & 86.42 & 87.82 \\
\hline
\end{tabular}

Three-variable Box-Behnken experimental design. with $y=\hat{y}+e$ and where $\hat{y}$ is the estimated response function; $y$, the measured response; $e$, the error, $b_{0}, b_{j}, b_{j k}$ and $b_{j j}$, the estimated model coefficients. $X_{j}$ are the coded variables which take the levels -1 and +1 when natural variables take their low and high levels, respectively. In this study, the generation and the data treatment of the Box-Behnken design are performed using the experimental design software NemrodW [23].

\subsection{Reaction products analysis}

\subsubsection{HPLC analysis}

In order to purify propyl gallate and to analyse the extracted polyphenols, we have used an analytical Shimadzu C-18 column composed of an LC-10ATvp pump and an SPD-10Avp detector. The used mobile phase was $0.1 \%$ formic acid in water $(A)$ versus $80 \%$ acetonitrile in water (B) for a total running time of $15 \mathrm{~min}$. The absorbance was measured at $275 \mathrm{~nm}$. The measurement of the area of the new peak appeared after esterification allows us to confirm the conversion yield obtained. About $100 \mu \mathrm{g}$ of purified propyl gallate was obtained after HPLC analysis described above (data not shown).

\subsubsection{FTIR and NMR analysis}

In order to check the esterification process, we used FTIR NEXUS spectrophotometer (Nicoleit, Madison, WIS, USA) showing the shift of the carbonyl of carboxylic acid group to the carbonyl of ester group. The sample was mixed with KBr. FTIR spectra were acquired after 32 scans between 4000 and $400 \mathrm{~cm}^{-1}$ with spectral resolution of $4 \mathrm{~cm}^{-1}$.

The structure of the propyl gallate ester was also confirmed by ${ }^{13} \mathrm{C}$ NMR (Madison, USA). Then, samples were dissolved in $\mathrm{CDCl} 3$ containing trace amounts of tetramethylsilane which was used as an internal chemical shift reference to indicate, in parts per million (ppm), the difference of the resonance frequency.

\subsection{Antioxidant activity in refined soy oil}

Propyl gallate, gallic acid, ascorbic acid and butylated hydroxyanisole (BHA) were dissolved $(1 \mathrm{mg} / \mathrm{mL})$ in ethanol and added to refined soy oil at $200 \mu \mathrm{g} / \mathrm{g}$ as previously described [24]. The phenowere stored in the dark at $70^{\circ} \mathrm{C}$. The stability of oil was evaluated by the measurement of the conjugated dienes $C D\left(K_{232}\right)$, triene formation $\mathrm{CT}\left(\mathrm{K}_{270}\right)$ and the peroxide value (PV). lic antioxidants were mixed with oil by stirring for 5 min. Samples 
Table 3

Analysis of variance.

\begin{tabular}{|c|c|c|c|c|c|}
\hline Source of variation & Sum of squares & Degrees of freedom & Mean square & Ratio & Significance \\
\hline Regression & 3991.72 & 9 & 443.525 & 40.867 & $0.0106^{* * *}$ \\
\hline Residuals & 65.11 & 6 & 10.853 & & \\
\hline Total & 4056.84 & 15 & & & \\
\hline$R^{2}$ & 0.984 & & & & \\
\hline
\end{tabular}

**** Significant at the level 99.9\%.

\subsubsection{Measurement of conjugated diene $(C D)$ and triene $(C T)$}

Conjugated diene (CD) and conjugated triene $(C T)$ are suited for measuring the oxidative state of oil [25]. Refined oil $(0.1 \mathrm{~g})$ stored at $70^{\circ} \mathrm{C}$ was dissolved in $25 \mathrm{~mL}$ of hexane and the absorbance was measured at 232 and $270 \mathrm{~nm}$, using hexane as a blank. All measurements were performed in duplicate.

\subsubsection{Measurement of peroxide value (PV)}

Peroxide value was determined according to the method of Low and $\mathrm{Ng}$ [26]. The oil sample (1 g) was dissolved in $25 \mathrm{~mL}$ of a chloroform and acetic acid mixture $(2 / 3, v / v)$. To this mixture, $1 \mathrm{~mL}$ of saturated potassium iodide solution was added followed by the addition of $75 \mathrm{~mL}$ of distilled water. The peroxide value was determined by titrating the iodine liberated from potassium iodide with standardised $0.01 \mathrm{~N}$ sodium thiosulfate solution using $0.5 \mathrm{~mL}$ of starch solution $(1 \%, \mathrm{w} / \mathrm{v})$ as indicator. The peroxide value was expressed as milli equivalents of free iodine per $\mathrm{kg}$ of lipid. All measurements were performed in duplicate.

\subsection{Antimicrobial activity}

Antimicrobial activity of propyl gallate and gallic was tested against Escherichia coli and S. xylosus using disc diffusion and broth macrodilution methods. Ampicillin was used as a standard reference. Propyl gallate and gallic acid were dissolved in ethanol at a final concentration of 21 and $17 \mathrm{mg} / \mathrm{mL}$, respectively. The solutions were sterilized by the microfilter sterile. One-hundred microlitres $\left(2 \times 10^{8} \mathrm{CFU} / \mathrm{mL}\right)$ of suspension were spread on the solid media and grown for $24 \mathrm{~h}$ then spread over the solid surface of nutrient agar medium. Filter paper discs ( $6 \mathrm{~mm}$ of diameter), loaded with propyl gallate, gallic acid and ampicillin were placed on the surface of nutrient agar and incubated at $37^{\circ} \mathrm{C}$ for $24 \mathrm{~h}$. Then, the diameters of inhibition zones were measured. All experiments were done in triplicates.

\subsubsection{Determination of minimum inhibitory concentration (MIC) and minimum bactericidal concentration (MBC)}

Minimum inhibitory concentration (MIC) and minimum bactericidal concentration $(\mathrm{MBC})$ were determined in $\mathrm{LB}$ broth using a macrobroth dilution method as described by the Clinical and Laboratory Standards Institute (CLSI) (2007). The final concentration of bacteria in each macrobroth dilution tube was approximately $6.8 \times 10^{6} \mathrm{CFU} / \mathrm{mL}$ of LB.

The MIC was defined as the lowest concentration of the phenolic compound that resulted in no visible growth after $24 \mathrm{~h}$ of incubation at $37^{\circ} \mathrm{C}$.

The MBC was defined as the lowest concentration of the propyl gallate or the gallic acid at which inoculated microorganism was completely killed. Ampicillin was used as a positive control. All experiments were done in triplicates.

\subsubsection{Influence of propyl gallate in bacteria viability}

Samples were withdrawn at selected time points and serial dilutions were performed in sterile LB liquid before these samples were spread onto LB agar medium in Petri dishes. Bacterial growth was followed by incubation for $24 \mathrm{~h}$ at $37^{\circ} \mathrm{C}$ and was determined by enumerating the number of viable cells by plating serial dilutions in the nutrient agar medium.

\section{Results and discussion}

\subsection{Optimization of conversion yield}

\subsubsection{Preliminary study}

We studied the ability of immobilized S. xylosus lipase, produced in our laboratory, to synthesize the propyl gallate. A preliminary study carried out with seven variables using the One Variable At a Time method (OVAT) [20] showed that three variables had significant effects on the response. These variables were the reaction temperature $\left(X_{1}\right)$, the amount of immobilized lipase $\left(X_{2}\right)$ and the 1-propanol: gallic acid molar ratio $\left(X_{3}\right)$. The levels of the three other variables having only a small effect on the esterification yield were fixed as follows: reaction time: $6 \mathrm{~h}$, molecular sieve: $0 \%$, stirring speed: $200 \mathrm{rpm}$. Hexane ( $2 \mathrm{~mL}$ ) was chosen as a suitable solvent for both gallic acid and propanol solubilization.

\subsubsection{Experimental design results}

3.1.2.1. Experimental conditions. In order to define the explored experimental domain, the level values of variables were chosen in such a way that their limits were as wide as possible while all those experiments were done (Table 1 ). The results are indicated in the matrix design (Table 2).

3.1.2.2. Model equation. The coefficients of the postulated model were calculated on the basis of the experimental responses (without including the checked points). The fitted model that expressed in coded variables was represented by the following equation.

$$
\begin{aligned}
\hat{y}= & 83.6+3.5 X_{1}+16.9 X_{2}+6.5 X_{3}-6.2 X_{1}^{2}-16.2 X_{2}^{2}-0.9 X_{3}^{2} \\
& -2.4 X_{1} X_{2}+X_{1} X_{3}-0.4 X_{2} X_{3}
\end{aligned}
$$

3.1.2.3. Analysis of variance and validation of the model. The good quality of the fitted model was attested with the analysis of the variance (ANOVA) as demonstrated in Table 3. Indeed, this table shows that the sum of squares related to the regression was statistically significant when using the $F$-test at a $99.9 \%$ probability level, which suggests that the variation accounted for by the model was significantly greater than the residual variation. Likewise, the coefficient of multiple determination of the polynomial model termed $R^{2}$, indicates that more than $98 \%$ of the variability in the response could be explained by the second-order polynomial predicted equation given above. In this respect, the validity of the model has been established by comparing the obtained results at the four check points (experiments 17-20) with the predicted values (Table 2). These results seem to confirm the validity of the model.

\subsubsection{Interpretation of the response surface model}

The coordinates of the stationary point of the isoresponse curves corresponding to a maximum of conversion yield are: temperature of the reaction, $52^{\circ} \mathrm{C}$; immobilized lipase, $400 \mathrm{IU}$; 1 -propanol/gallic acid molar ratio, 160 . 

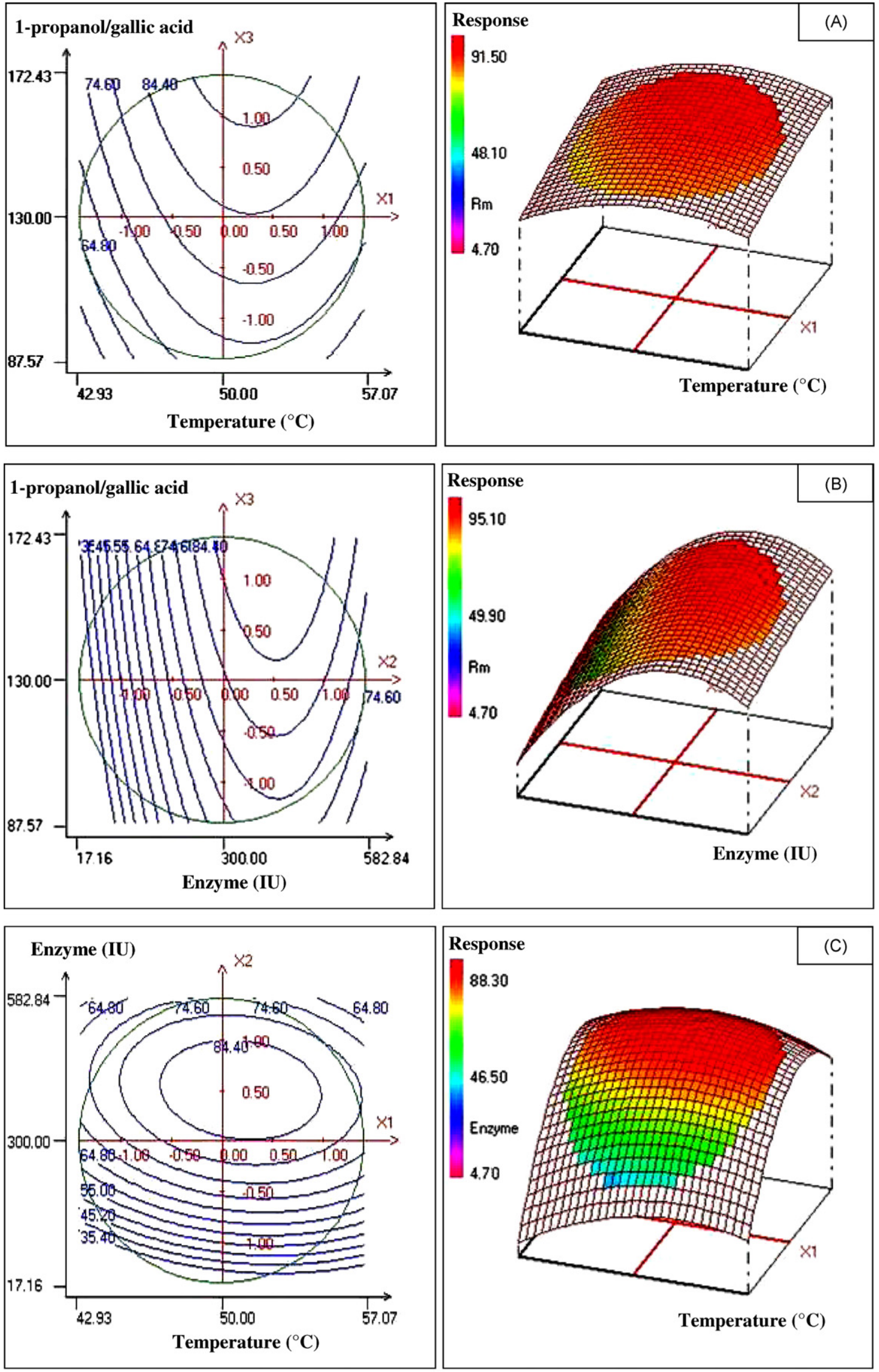

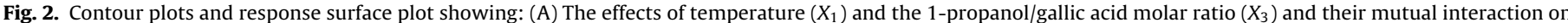

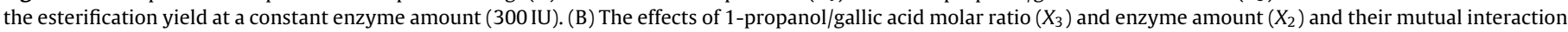

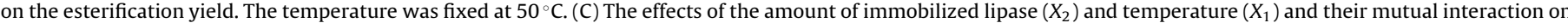
the esterification yield at a constant 1-propanol/gallic acid molar ratio of 160 . 


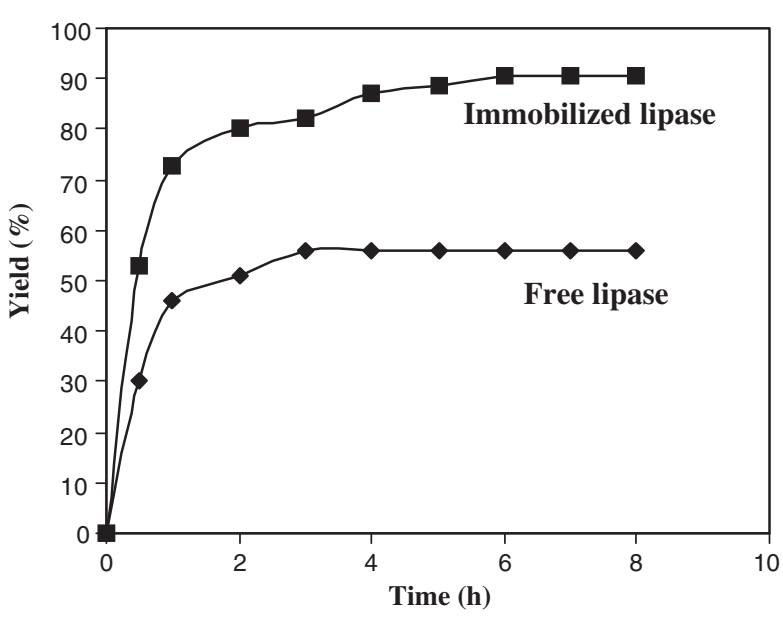

Fig. 3. The time course of propyl gallate synthesis using free and immobilized lipase. The experimental conditions were: $400 \mathrm{IU}$ of enzyme, 1-propanol/gallic acid molar ratio of 160 dissolved in $2 \mathrm{~mL}$ hexane. The reaction mixture was stirred at $200 \mathrm{rpm}$ at $52{ }^{\circ} \mathrm{C}$. A control without enzyme was carried out under the same condition. Experiments were carried out in triplicates.

The response surfaces and the isoresponse curves are plotted in function of two factors while the third was maintained constant at its main level (Fig. 2A-C). These figures show that the conversion yield was highly enhanced $(90 \%)$ by increasing the 1 propanol/gallic acid molar ratio (over 160). These results are in agreement with those obtained by Xiao and Yong who stipulate that the stability of lipases is strongly affected when gallic acid is used as substrate [27]. Also, the contour plots in Fig. 2B and C emphasize the important role of temperature and quantity of enzymes. Indeed, the conversion yield decreased dramatically at a low temperature. Also, high enzyme levels (above $400 \mathrm{IU}$ ) decreased the conversion yield dramatically. This result can be explained by the fact that at high amount of lipase, the molecules of the enzyme can aggregate together and the active site cannot be exposed to the substrates.

In order to confirm these results, three independent, complementary experiments were carried out under the optimal conditions (coordinates of the stationary point given above). Indeed, the time course of the propyl gallate synthesis by free and immobilized S. xylosus lipase was made under optimal conditions (Fig. 3). From the presented data, one can note that the conversion yield of the propyl gallate synthesis increases rapidly to reach its maximum $(90 \pm 3.5 \%)$ after $4 \mathrm{~h}$ of incubation time. Thus,
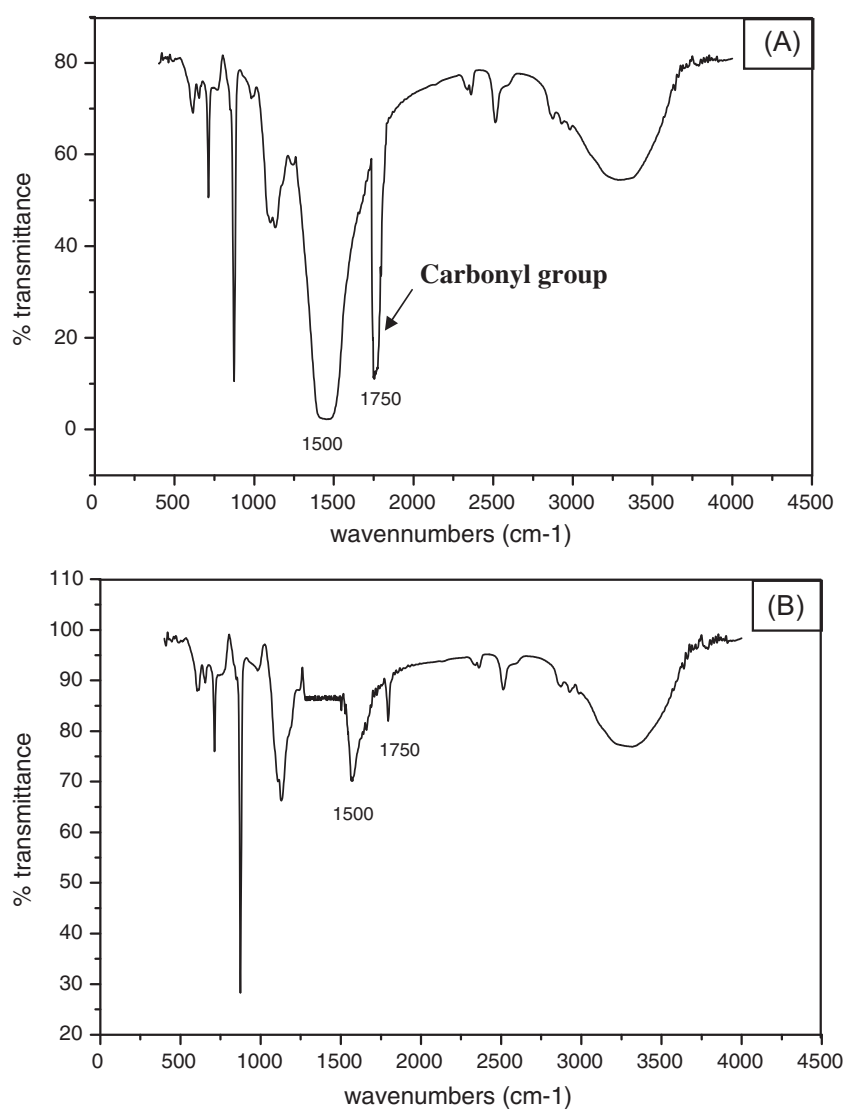

Fig. 5. FTIR spectra of the propl gallate adsorbed to $\mathrm{CaCO}_{3}$ before (A) and after (B) the wash step by the ethanol. The carbonyl group of the ester was marked by the arrow.

the obtained experimental conversion yield was very close to the predicted value estimated (96.86 $\pm 3.70 \%)$. Noteworthy, the conversion yield of the obtained propyl gallate synthesis when using immobilized SXL, is higher than that obtained by free lipase. This result is also in agreement with that obtained by Ghamgui et al. [28] and Horchani et al. [29] who state that the immobilization of staphylococcal lipases into $\mathrm{CaCO}_{3}$ increases both their stability at high temperature and their ability to tolerate the presence of organic solvents. In addition, one can relate the low conversion yield obtained with free enzyme and its inactivation at the
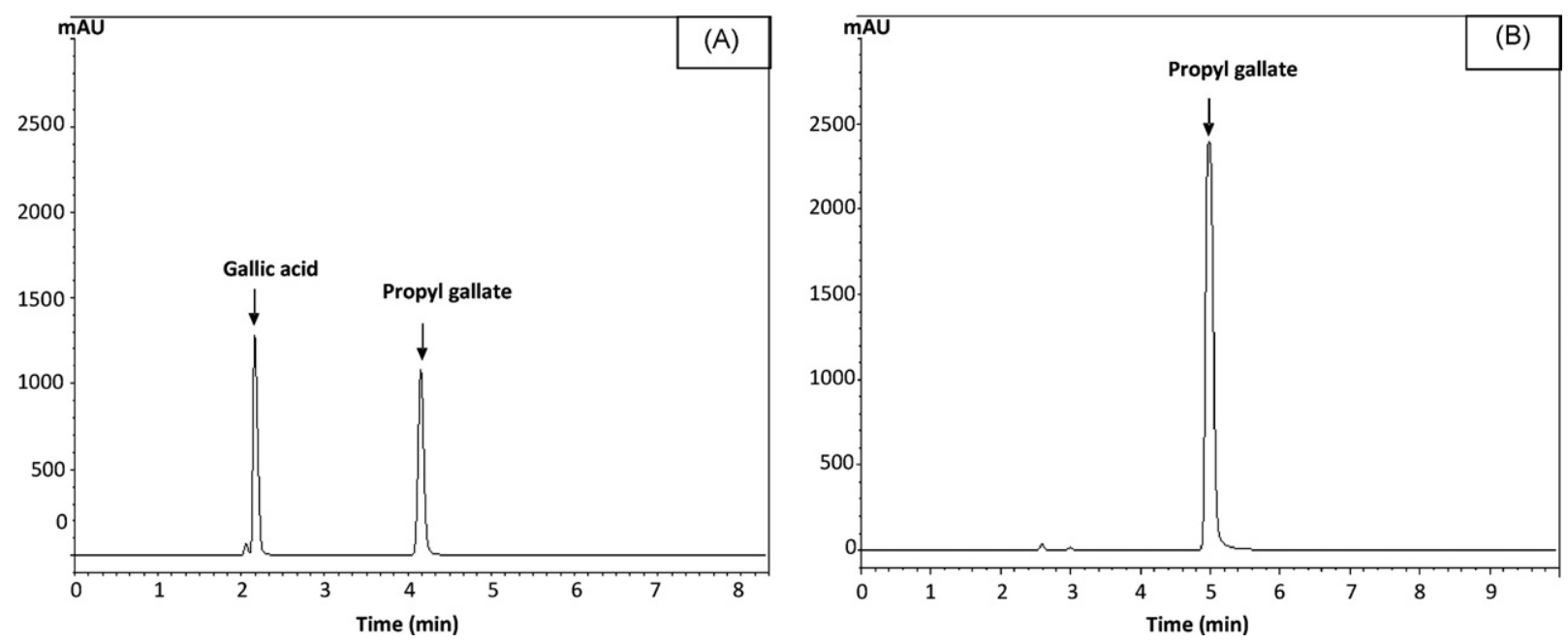

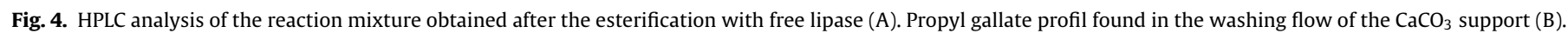


interface of the biphasic media. In order to confirm these findings, a reaction mixture was carried out using free lipase at different concentrations of molecular sieve (data not shown). An increase in the conversion yield was observed reaching $70 \%$ after $4 \mathrm{~h}$ of incubation.

\subsection{Analysis of the reactions products}

\subsubsection{HPLC analysis}

The HPLC analysis of the reaction mixture obtained after esterfification (Fig. 4) shows paradoxically the presence of propyl gallate only when free lipase is used (Fig. 4A). This apparent contradiction could be related to the adsorption of the ester to $\mathrm{CaCO}_{3}$. To confirm this hypothesis, the reaction mixture was removed by centrifugation and the $\mathrm{CaCO}_{3}$ was washed twice with $1 \mathrm{~mL}$ of ethanol. The HPLC analysis of the washing flow showed the presence of a single peak corresponding to propyl gallate (Fig. 4B) emerging at the same time as the pure commercial propyl gallate used as reference.

\subsubsection{FTIR measurement and ${ }^{13}$ C NMR}

The chemical structure of propyl gallate was confirmed by FTIR by analyzing the $\mathrm{CaCO}_{3}$ before (Fig. 5A) and after (Fig. 5B) being washed by ethanol. Typical stretches at $1750 \mathrm{~cm}^{-1}$ and at $1500 \mathrm{~cm}^{-1}$ corresponding to the carbonyl ester and the aromatic groups, respectively, were observed before $\mathrm{CaCO}_{3}$ washing with ethanol. The intensity of these bands decreased significantly after the wash step with ethanol. This result confirms the fact that the propyl gallate was synthesized successfully and was adsorbed to $\mathrm{CaCO}_{3}$. The same result was observed after ${ }^{13} \mathrm{C}$ NMR analysis of the reaction mixture before and after esterification (data not shown), showing that the intensity of the band appeared at $168.6 \mathrm{ppm}$, which corresponds the carbonyl group of the synthesized ester, decreased significantly after washing $\mathrm{CaCO}_{3}$ with ethanol (data not shown).

\subsection{Antioxidant activity}

In order to determine its antioxidant power, the propyl gallate was added to the refined soy oil at $200 \mu \mathrm{g} / \mathrm{g}$ and the results were compared to BHA with ascorbic acid and gallic acid used as standard antioxidants. The oxidation of refined oil, stored at $70^{\circ} \mathrm{C}$, was monitored by measuring conjugated diene (CD) and triene formation (CT) (Fig. 6A and B). The conjugated diene, measured at $232 \mathrm{~nm}$, determines the primary product of oil oxidation. The conjugated triene, measured at $270 \mathrm{~nm}$, enquires the secondary oxidation products such as aldehydes and ketones, and hence may be an indication of the formation of the final products of oil peroxidation. From the data presented in Fig. 6A and B, a significant increase was observed in $\mathrm{CD}$ and $\mathrm{CT}$ for controlled refined soy oil. The addition of antioxidants exhibited prominent antioxidant activity. Results indicated clearly that the propyl gallate shows a high antioxidant activity compared to that of BHA (Fig. 6A and B). So, the diminution of the absorbance at $232 \mathrm{~nm}$ and $272 \mathrm{~nm}$ due to the formation of conjugated diene (CD) and triene, after the addition of propyl gallate, confirms the high protective effect of propyl gallate against oxidation of refined soy oil.

The antioxidant power of propyl gallate was also tested by measuring the peroxide value after adding various concentrations of
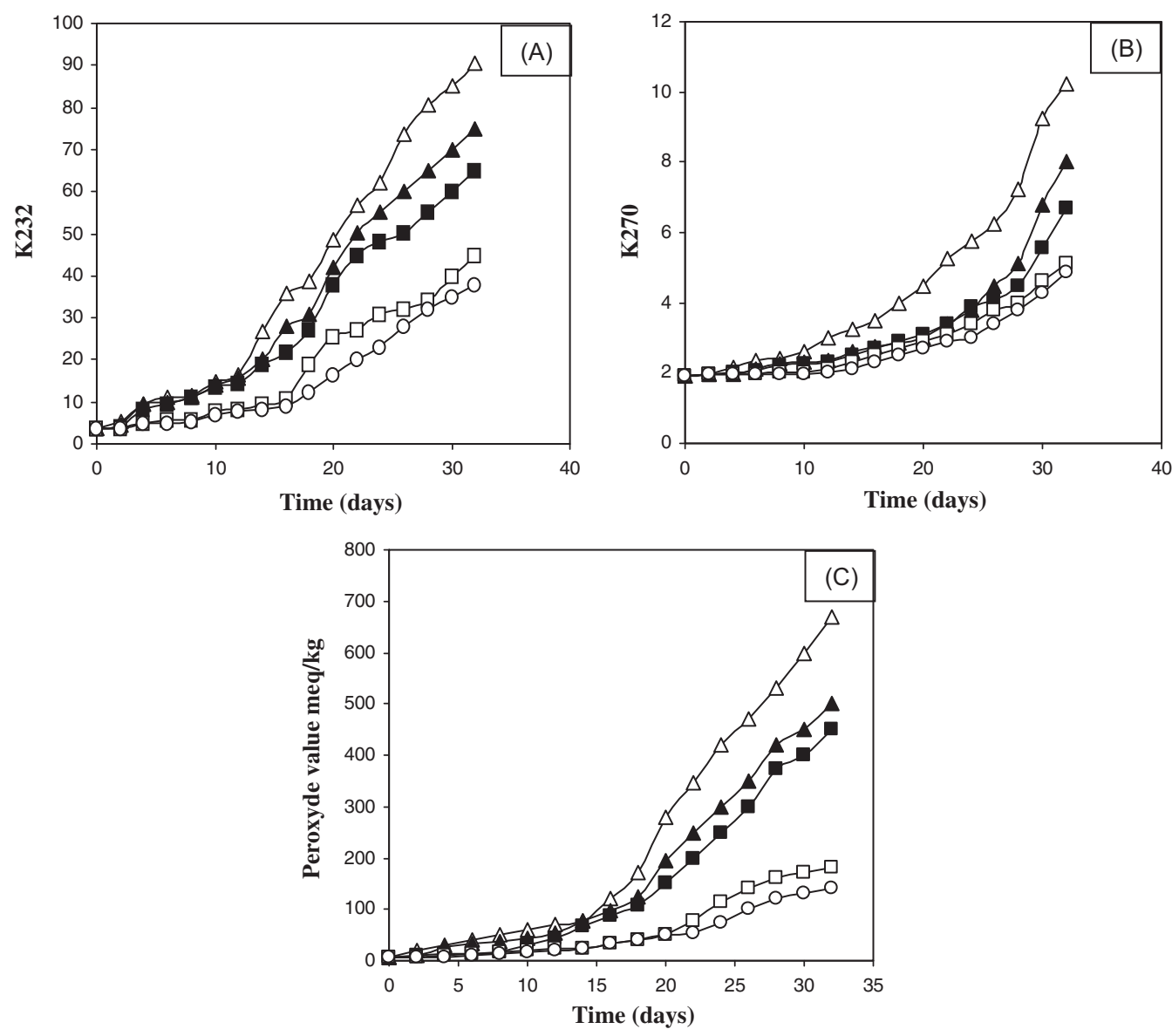

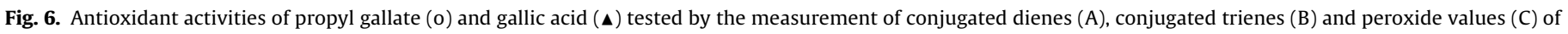
refined soy oil during storage at $70^{\circ} \mathrm{C}$. For each test, negative $(\Delta)$ and positive controls using BHA ( $\square$ ), ascorbic acid $(\boldsymbol{\square})$ were run in parallel. 
Table 4

Antimicrobial activity of the propyl gallate, gallic acid and ampicillin.

\begin{tabular}{|c|c|c|c|c|c|c|c|c|c|}
\hline \multirow[t]{2}{*}{ Microorganisms } & \multicolumn{3}{|c|}{ Propylgallate } & \multicolumn{3}{|c|}{ Gallic acid } & \multicolumn{3}{|c|}{ Ampicillin } \\
\hline & $\mathrm{DD}^{\mathrm{a}}$ & $\mathrm{MIC}^{\mathrm{b}}$ & $\mathrm{MBC}^{\mathrm{b}}$ & $\mathrm{DD}^{\mathrm{a}}$ & $\mathrm{MIC}^{\mathrm{b}}$ & $\mathrm{MBC}^{\mathrm{b}}$ & $\mathrm{DD}^{c}$ & $\mathrm{MIC}^{\mathrm{b}}$ & $\mathrm{MBC}^{\mathrm{b}}$ \\
\hline Staphylococcus xylosus & 31 & 1 & 1 & 24 & 1 & 2 & 35 & 0.02 & 0.05 \\
\hline Staphylococcus aureus & 28 & 2 & 3 & 22 & 2 & 4 & 30 & 0.2 & 0.2 \\
\hline Escherichia coli & 36 & 0.5 & 0.5 & 25 & 1 & 2 & 20 & 0.02 & 0.04 \\
\hline
\end{tabular}

DD diameter of zone of inhibition ( $\mathrm{mm}$ ) including disc diameter of $6 \mathrm{~mm}$.

a Tested at concentration of $1.5 \mathrm{mg} / \mathrm{disc}$.

b Values given as $\mathrm{mg} / \mathrm{ml}$.

c Tested at concentration of $10 \mu \mathrm{g} /$ disc.

propyl gallate (Fig. 6C). In fact, the measurement of the primary oil oxidation is widely used and it indicates the amount of peroxides formed in fats and oil during oxidation [30]. Changes in peroxide values are presented in Fig. 6C. Peroxide value of the controlled refined soy oil increased from 6 to $670 \mathrm{meq} / \mathrm{kg}$ after 32 days of incubation at $70^{\circ} \mathrm{C}$. After adding $300 \mathrm{ppm}$ of propyl gallate, the peroxide values decreased significantly to reach $115 \mathrm{meq} / \mathrm{kg}$. The analytical investigations we have explained so far reveal that the synthesized propyl gallate is an excellent antioxidant for refined soy oil. Its activity is comparable to that of BHA and much higher than that of ascorbic acid, both of which are used as standard antioxidants.

\subsection{Antimicrobial activity}

The antimicrobial activity of the propyl gallate and gallic acid was evaluated against Gram-positive (S. xylosus and S. aureus) and Gram-negative (E. coli) bacteria using disc diffusion and broth macrodilution methods. The disc diameters of the inhibition zone (DD), the minimum inhibitory concentrations (MIC) and the minimum bactericidal concentration (MBC) of both the propyl gallate and gallic acid against the susceptible pathogenic organisms are shown in Table 4 . The antimicrobial activity was compared to ampicillin, a currently used antibiotic of broader spectrum (Grampositive and Gram-negative bacteria). According to the results presented in Table 4, one can note that propyl gallate as well as gallic acid showed a good inhibitory effects against Gram-positive and Gram-negative bacteria species being tested. The diameters of the inhibition zone of the newly synthesized propyl gallate $(1.5 \mathrm{mg})$ were: $36 \mathrm{~mm}$ for E. coli, $31 \mathrm{~mm}$ for S. xylosus and $28 \mathrm{~mm}$ for S. aureus

The MIC values of propyl gallate were on the range of $1 \mathrm{mg} / \mathrm{mL}$ on $S$. xylosus and $0.5 \mathrm{mg} / \mathrm{mL}$ on E. coli. We found also that gallic acid and propyl gallate showed the same MIC values against Grampositive bacteria. Yet, the propyl gallate showed better inhibitory effects on Gram-negative bacteria (MIC $=500 \mu \mathrm{g} / \mathrm{mL}$ ) than the gallic acid $(\mathrm{MIC}=1000 \mu \mathrm{g} / \mathrm{mL})$

The highest effect of propyl gallate on Gram-negative more than on Gram-positive bacteria is probably due to the more complex cell wall structure of the Gram-positive bacteria which has additional lipopolysaccharides on the outer surface, which generally reduces the ability of most antibiotics and extracts to penetrate the bacterial cells [31].

The bacterial effect of propyl gallate against S. xylosus was confirmed by the time kill curve experiment (data not shown). Cultures of a Gram-positive (S. xylosus) and a Gram-negative (E. coli), with a cell density of $6.8 \times 10^{6} \mathrm{CFU} / \mathrm{mL}$, were exposed to different concentrations of propyl gallate. The number of viable cells was determined after different periods of incubation. It was found that propyl gallate reduced the number of viable Gram-positive or Gram-negative cells rapidly and within the $1 \mathrm{~h}$. No viable cells were detected after being exposed to $1 \mathrm{mg} / \mathrm{mL}$ of propyl gallate. In fact it has been reported that propyl gallate inhibits the growth of microorganisms by blocking respiration and nucleic acid synthesis [32]. This antibacterial activity of the hydrophobic propyl gallate molecule is probably due to its increased interaction with the cytoplasmic membrane, which results in the inhibition of the oxygen consumption and the disruption of the membrane located in the respiratory chain [33].

\section{Acknowledgments}

This work is a part of a doctoral thesis by Ahlem BOUAZIZ whose research was supported financially by "Ministère de l'Enseignement Supérieur et de la Recherche scientifique-Tunisia" through a grant to "Laboratoire de Biochimie et de Génie Enzymatique des Lipases-ENIS".

\section{References}

[1] J. Karovicova, P. Simko, J. Chromatogr. A 882 (2000) 271-281.

[2] D.B. Clayson, F. Iversion, E. Nera, E. Lok, C. Rogers, C. Rodrigues, Food Chem. Toxicol. 24 (1986) 1171-1182.

[3] N. Ito, M. Hirose, S. Fukushima, H. Tsuda, T. Shirai, H. Tatematsu, Food Chem. Toxicol. 24 (1986) 1071-1082.

[4] J.W. Daniel, Xenobiotica 16 (1986) 1073-1078.

[5] J.C. Dacre, Food Cosmet. Toxicol. 12 (1974) 125-129.

[6] T.W. Wu, K.P. Funy, L.H. Zeny, J. Wu, H. Nakamura, Biochem. Pharmacol. 48 (1994) 419-422.

[7] P.M. Rosin, F. Stich, Cancer Lett. 9 (1980) 299-305.

[8] K.M. Abdo, J.E. Huff, J.K. Haseman, C.J. Alden, Food Chem. Toxicol. 24 (1986) 1091-1097.

[9] J.R. Reddan, F.J. Giblin, M. Sevilla, V. Padgaonkar, C.D. Dziedzic, R.V. Leverenz, C.I. Misra, S.J. Chang, T.J. Pena, Exp. Eye Res. 76 (2003) 49-59.

[10] S. Raghavan, H.J. Hultin, Food Biochem. 33 (2005) 163-175.

[11] M. Hirose, S. Takahashi, K. Ogawa, M. Futakuchi, T. Shirai, Food Chem. Toxicol. 37 (1999) 985-992.

[12] K. Karthikeyan, B.R. Sarala Bai, K. Gauthaman, S. Niranjali Devaraj, J. Pharm. Pharmacol. 57 (2005) 67-73.

[13] W.K. Jeon, B.C. Kim, Biochem. Biophys. Res. Commun. 361 (2007) 645-650.

[14] H. Horchani, M. Chaâbouni, Y. Gargouri, A. Sayari, Carbohydr. Polym. 79 (2010) $466-474$.

[15] Y. Gargouri, R. Julien, A. Sugihara, L. Sarda, R. Verger, Biochim. Biophys. Acta 795 (1984) 326-331.

[16] H. Nawaz, J. Shi, G. Mittal, Y. Kukudo, Sep. Purif. Technol. 48 (2006) 176-181.

[17] V. Nwuha, J. Food Eng. 44 (2000) 233-238.

[18] X. Wu, J. Li, X. Li, C.L. Hsieh, P.M. Burgers, M.R. Lieber, Nucleic Acids Res. 24 (1996) 2036-2043.

[19] L.A. Sarabia, M.C. Ortiz, Chem. Biochem. Data Anal. 1 (2009) 345-390.

[20] R. Carlson, Design and Optimization in Organic Synthesis, New York, 1992, pp. 26-27.

[21] J. Goupy, Plan d'expériences pour surface de réponses, DUNOD, Paris, 1999, pp. 253-293.

[22] G.A. Lewis, G.A. Mathieu, T.R. Phan, Pharmaceutical Experimental Design, Marcel Dekker Inc., New York, 1999.

[23] D. Matheiu, J. Nony, T.R. Phan, NEMROD-W Software, LPRAI, Marseille, 2000.

[24] W. Della, Y. Wong, C. Mak, C. Sze, W. Yao, J. Food Compos. Anal. 19 (2006) 784-791.

[25] L. McGinely, in: J.B. Rossel, J.L.R. Printed (Eds.), Analysis of Oil Seeds, Fat and Fatty Food, Elsevier, Applied Science, New York, 1991, pp. 440-470.

[26] L.K. Low, C.S. Ng, in: H. Hasegawa (Ed.), Laboratory Manual on Analytical Methods and Procedures for Fish and Fish Products, Marine Fisheries Research Department, Southeast Asian Fisheries Development Center, Singapore, 1978, pp. C7.1-C7.3.

[27] W.Y. Xiao, Q.L. Yong, J. Mol. Catal. B: Enzyme 40 (2006) 44-50. 
[28] H. Ghamgui, M. Karra-Châabouni, Y. Gargouri, Enzyme Microb. Technol. 35 (2004) 355-363.

[29] H.Horchani, S. Ouertani, Y. Gargouri, A. Sayari, J. Mol. Catal. B: Enzyme 61 (2009) 194-201.
[30] O. Gulcan, H. Bedia, J. Food Eng. 79 (2007) 1391-1396.

[31] H.P. Rang, M. Dale, Pharmacology, Churchill, Edinburgh, 1987.

[32] I. Boyd, E.G. Beveridge, Microscopy 24 (1979) 173-184.

[33] I. Kubo, P. Xiao, K. Fujita, Bioorg. Med. Chem. Lett. 12 (2002) 113-116. 